\title{
Tempo de cozimento e textura de raízes de mandioca
}

\author{
Cooking time and texture of cassava roots
}

\section{Autores | Authors}

Simone Vilela TALMA

Universidade Estadual do Norte Fluminense Darcy Ribeiro (UENF) Centro de Ciências e Tecnologias Agropecuárias (CCTA) Laboratório de Tecnologia de Alimentos (LTA)

Campos dos Goytacazes/RJ - Brasil e-mail: simonevtalma@yahoo.com.br

\section{$\triangle$ Selma Bergara ALMEIDA}

Universidade Estadual do Norte Fluminense Darcy Ribeiro (UENF) Centro de Ciências e Tecnologias Agropecuárias (CCTA) Laboratório de Tecnologia de Alimentos (LTA) Av. Alberto Lamego, 2000 Parque Califórnia CEP: 28013-602 Campos dos Goytacazes/RJ - Brasil e-mail: sbergara@uenf.br

\section{Rozana Moreira Pereira LIMA}

Universidade Estadual do Norte Fluminense Darcy Ribeiro (UENF) Centro de Ciências e

Tecnologias Agropecuárias (CCTA) Laboratório de Engenharia Agrícola (LEAG) Campos dos Goytacazes/RJ - Brasil e-mail: rozalim24@yahoo.com.br

\section{Henrique Duarte VIEIRA}

Universidade Estadual do Norte Fluminense Darcy Ribeiro (UENF) Centro de Ciências e Tecnologias Agropecuárias (CCTA) Laboratório de Fitotecnia (LFIT) Campos dos Goytacazes/RJ - Brasil e-mail: henrique@uenf.br

\section{Pedro Amorim BERBERT}

Universidade Estadual do Norte Fluminense Darcy Ribeiro (UENF) Centro de Ciências e

Tecnologias Agropecuárias (CCTA) Laboratório de Engenharia Agrícola (LEAG) Campos dos Goytacazes/RJ - Brasil e-mail: pberbert@uenf.br

Autor Correspondente / Corresponding Author

Recebido / Received: 15/05/2012 Aprovado / Approved: 08/05/2013 Publicado / Published: jun./2013

\section{Resumo}

O objetivo deste trabalho foi avaliar a adequação de medidas instrumentais de textura como índice de qualidade de raízes da mandioca de mesa e sua correlação com o tempo de cozimento. Quinze raízes de mandioca foram colhidas no $11 .{ }^{\circ}$ mês de cultivo na região noroeste fluminense. Pedaços de raízes foram cozidos em água, sendo o tempo de cozimento determinado, em triplicata, quando se observou pouca resistência à penetração do garfo. A resistência ao corte foi realizada nas polpas cruas e cozidas, em cinco a nove repetições, operando o texturômetro TA. XT Plus Texture Analyser com probe Warner-Bratzler Blade HDP/BSW, velocidades de pré-teste de $0,2 \mathrm{~cm} / \mathrm{s}$, de pós-teste e de teste de $0,5 \mathrm{~cm} / \mathrm{s}$, e distância de $5 \mathrm{~cm}$. Os dados foram analisados por ANOVA e teste de média Tukey (tempo de cozimento); GLM, LSMEANS e PDIFF (resistência ao corte) e análise de correlação de Pearson $(p \leq 0,05)$. A variedade Viçosa Martinha destacou-se por apresentar o menor tempo de cozimento (18 minutos) e menores valores de resistência ao corte das polpas crua $(10,6 \mathrm{~N})$ e cozida $(0,7 \mathrm{~N})$. Com exceção das variedades Aipim Pretinho, IAC Espeto e IAC 13, todas as demais podem ser consideradas adequadas como mandiocas de mesa por apresentarem tempos de cozimento iguais ou inferiores a 30 minutos. Verificou-se correlação significativa entre a resistência ao corte da polpa cozida e o tempo de cozimento $(0,62)$, porém moderada, em razão dos altos valores de coeficiente de variação observados para as medidas de textura, evidenciando a heterogeneidade das raízes. Portanto, a determinação do tempo de cozimento, nas condições deste experimento, é mais adequada como índice da qualidade de raízes de mandioca associado à textura.

Palavras-chave: Manihot esculenta; Cocção; Resistência ao corte; Medida instrumental.

\section{Summary}

The objective of this work was to evaluate the adequacy of instrumental texture analyses as a quality index for cassava roots for direct consumption, and the correlation with cooking time. Fifteen cassava roots were harvested in the eleventh month of growth in the northwest of the State of Rio de Janeiro. Pieces of roots were boiled in water and the cooking time determined in triplicate, to the point where there was little resistance to penetration by a fork. The shear strength was determined in the raw and cooked pulps with five to nine replicates, using the texturometer TA-XT Plus Texture Analyser with the Warner-Bratzler Blade HDP / BSW probe, a pre-test speed of $0.2 \mathrm{~cm} / \mathrm{s}$, post-test and test speed of $0.5 \mathrm{~cm} / \mathrm{s}$ and distance of $5 \mathrm{~cm}$. The data were analyzed by ANOVA and Tukey (cooking time), GLM, LSMEANS and PDIFF (shear resistance) and Pearson's correlation analysis $(p \leq 0.05)$. The variety Viçosa Martinha stood out due to its shorter cooking time (18 minutes) and lower shear resistance values for the raw $(10.6 \mathrm{~N})$ and cooked $(0.7 \mathrm{~N})$ pulps. With the exception of the varieties Aipim Pretinho, IAC 13 and IAC Espeto, all the others could be considered suitable for direct consumption since they presented cooking times equal or below 30 minutes. There was significant correlation between the shear strength of the cooked pulp and the cooking time (0.62), although moderated by the high values obtained for the coefficient of variation of the texture measurements, highlighting the heterogeneity of the roots. Thus the determination of the cooking time under the experimental conditions used here is more suitable as a quality index of cassava roots associated with the texture.

Key words: Manihot esculenta; Cooking; Shear resistance; Instrumental measurement. 


\section{Introdução}

A mandioca (Manihot esculenta Crantz) está presente nas diversas regiões do mundo, por apresentar tolerância às condições adversas de clima e solo, além de suas raízes serem das mais importantes fontes de carboidratos e de subsistência para as populações mais carentes (FOLEGATTI e MATSUURA, 1997; LORENZI, 2003; OSIRU et al., 1992; MATTOS et al., 1979). Em 2010, a produção anual brasileira de raízes de mandioca foi em torno de 24,3 milhões de toneladas (FAO, 2010).

Um dos fatores de interesse dentro do conceito de qualidade culinária de raízes de mandioca de mesa, frequentemente abordado nos estudos sobre esse produto, é o tempo de cozimento: quanto menor, melhor a qualidade da massa gerada. Considera-se tempo ótimo entre 15 e 25 minutos, e raiz de baixa qualidade, quando ultrapassa 30 minutos (LORENZI 1994; WHEATLEY e GOMEZ, 1985).

Alguns pesquisadores consideram que, para a mandioca de mesa, a polpa cozida deve ser facilmente esmagada e desfeita, quando amassada com um garfo, formando uma pasta de textura farinácea, consistência plástica e moldável (BORGES et al., 1992; WHEATLEY, 1987). Na verdade, essas características de textura são desejáveis ou não, conforme a finalidade de uso da raiz. Se consumida como escondidinho, purê, sopa ou bolinho, quanto mais macia a raiz, melhor sua qualidade; se consumida como mandioca frita, cozida com sal ou com açúcar ou com manteiga, as características de textura adequadas devem ser verificadas a partir de estudos com consumidores, não encontrados na literatura até o momento.

De qualquer forma, essas variáveis possuem oscilações entre raízes de uma mesma cultivar em função do tipo de solo, da variedade e da idade da planta (BORGES et al., 1992; FUKUDA e BORGES, 1990, 1988); BORGES e FUKUDA, 1989).

Por outro lado, estudos têm sido realizados utilizando-se métodos instrumentais de análise de textura por constituir uma alternativa objetiva na avaliação dos alimentos, em substituição a outras metodologias de naturezas mais subjetivas ou empíricas, bem como para predizer mudanças químicas e de qualidade (LAWLESS e HEYMANN, 1999; FENNEMA, 1992). Estes métodos avaliam propriedades mecânicas a partir de forças aplicadas ao alimento, tais como compressão, cisalhamento, corte e tensão (LAWLESS e HEYMANN, 1999). Contudo, não foram encontrados registros da avaliação da correlação de medidas instrumentais de textura e de tempo de cozimento em raízes de mandioca.

Padonou et al. (2005) avaliaram a qualidade de 20 raízes de mandioca que foram cultivadas, cozidas e estudadas em Niaouli (Benin) quanto à firmeza em teste de penetração, utilizando Texture Analyser (Stevens-LFR), equipado com um cone de penetração movendo-se a $2 \mathrm{~mm} / \mathrm{s}$ para a profundidade de penetração final de $3 \mathrm{~mm}$. Diante dos resultados obtidos, os autores relataram que a propriedade de firmeza pode estar relacionada com propriedades funcionais do amido (como a viscosidade aparente após o cozimento), com o teor de cianeto e a quantidade de água absorvida após o cozimento das raízes. No entanto, pesquisas ainda devem ser realizadas com o intuito de esclarecer tais informações e, em particular, as respectivas funções da parede celular e as características do amido.

Menoli e Beleia (2007) utilizaram a medida instrumental para verificar as modificações da textura, como a compressão e o cisalhamento de raízes de mandioca da variedade Catarina Amarela, colhidas aos 8 meses após o plantio e submetidas a tratamento de pré-cozimento. Nesse estudo, amostras de mandioca foram mantidas às temperaturas controladas de $55^{\circ} \mathrm{C}$, $65^{\circ} \mathrm{C}$ e $75^{\circ} \mathrm{C}$ por uma hora em banho-maria e, em seguida, levadas a cozimento em água fervente a $98^{\circ} \mathrm{C}$. Foram utilizados texturômetro TA-TX2i Texture Analyzer, operando na medida de força de compressão com deformação de $15 \%$ da amostra com Probe P 0,5 e velocidades de pré-teste e pós-teste de $5 \mathrm{~mm} / \mathrm{s}$. A força de cisalhamento foi determinada com Probe Warner Bratzler HDP/BSK. A avaliação da textura permitiu verificar que os pré-tratamentos afetaram as estruturas intra e intercelulares das raízes, provocando a redução das forças de compressão e cisalhamento de acordo com o aumento da temperatura de pré-tratamento.

Diante disso, o objetivo deste trabalho foi avaliar a adequação de medidas instrumentais de textura como índice de qualidade de raízes da mandioca de mesa e sua correlação com o tempo de cozimento.

\section{Material e métodos}

As 15 variedades de raízes de mandioca (Manihot esculenta Crantz) estudadas neste trabalho já foram identificadas, algumas das quais foram lançadas pela Empresa Brasileira de Pesquisa Agropecuária (BR Eucalipto, BR Rosinha e BR Gema de Ovo), pelo Instituto Agronômico de Campinas (IAC 12, IAC 15, IAC Caapora, IAC Espeto e IAC 13), pela Universidade Federal de Viçosa (Cacau Violeira e Viçosa Martinha), pela Empresa de Pesquisa Agropecuária do Estado do Rio de Janeiro (Pesagro) e as demais eram de cultivo regional (Aipim Pretinho, Vermelho Alagoano, Amarelo Barcelos e Zumbi).

Raízes de duas plantas de cada variedade de mandioca estudada foram colhidas aleatoriamente e avaliadas no 11. ${ }^{\circ}$ mês após o plantio (out./2011). As raízes foram provenientes de cultivo na Estação Experimental da UENF-RJ, na ilha Barra do Pomba, no município de Itaocara, Rio de Janeiro, localizado na região noroeste 
fluminense, a $21^{\circ} 39^{\prime} 12^{\prime \prime}$ de latitude sul, $42^{\circ} 04^{\prime} 36^{\prime \prime}$ de longitude oeste e a $60 \mathrm{~m}$ de altitude (FONTES, 2002).

Em seguida, foi realizada a classificação das raízes, segundo critério praticado pela Companhia de Entrepostos e Armazéns Gerais de São Paulo (CEAGESP, 2011): tamanho entre 4 e 7 cm de diâmetro da raiz, visando à padronização do material utilizado neste trabalho.

Após a classificação, as raízes foram lavadas em água corrente, higienizadas em solução aquosa de hipoclorito de sódio (1 mL. $\left.\mathrm{L}^{-1}\right)$ e descascadas. Para a determinação do tempo de cozimento, as raízes foram cortadas em pedaços de $3 \mathrm{~cm}$ de comprimento longitudinal, preservando o diâmetro da raiz como peculiaridade de cada variedade.

O tempo de cozimento foi determinado, em três repetições, após o início da fervura da água $\left(98{ }^{\circ} \mathrm{C}\right)$, tendo sido colocada a proporção em massa de 1:10 (mandioca:água). As mandiocas foram consideradas cozidas quando ofereceram pouca resistência à penetração pelo garfo perpendicularmente em relação ao comprimento, sendo verificadas sempre pelo mesmo pesquisador (adaptado de BORGES et al., 2002).

A determinação da resistência ao corte foi realizada em cinco a nove repetições, em pedaços de $3 \mathrm{~cm}$ de comprimento, cortados ao meio no sentido longitudinal, de raízes de mandioca, das polpas cruas e cozidas de cada variedade. A variação do número de repetições das medidas foi em função da disponibilidade de raízes comerciais de cada variedade. Foi utilizado texturômetro TA.XT Plus Texture Analyser, operando com medida de força em compressão e probe Warner-Bratzler Blade HDP/
BSW, velocidades de pré-teste de $0,2 \mathrm{~cm} \cdot \mathrm{s}^{-1}$, de pós-teste e teste de $0,5 \mathrm{~cm} \cdot \mathrm{s}^{-1}$, e distância de $5 \mathrm{~cm}$.

Os valores de resistência ao corte foram analisados estatisticamente utilizando-se os procedimentos GLM (General Linear Models), LSMEANS e PDIFF ( $p \leq 0,05$ ), do programa estatístico SAS - Statistical Analysis System, versão 9.2. Os dados de tempo de cozimento foram submetidos à analise de variância (ANOVA) e ao teste de médias Tukey $(p \leq 0,05)$. A análise de correlação de Pearson $(p \leq 0,05)$ foi realizada entre as medidas de tempo de cozimento e resistência ao corte, bem como entre os valores de coeficiente de variação e o número de repetições das medidas de resistência ao corte. Esta última análise foi efetuada para verificar se os altos valores de coeficiente de variação eram resultantes do número de repetições das medidas ou da heterogeneidade das raízes.

\section{Resultados e discussão}

A Tabela 1 apresenta os resultados obtidos na determinação do tempo de cozimento e da resistência ao corte das diferentes raízes de mandioca.

Dentre as variedades estudadas, Zumbi, Viçosa Martinha, BR Gema de Ovo e BR Rosinha destacaram-se por apresentar o menor tempo de cozimento, não diferindo entre si (Tabela 1). Por outro lado, as variedades Aipim Pretinho, IAC Espeto e IAC 13 apresentaram tempos de cozimento iguais ou superiores a 30 minutos, considerados inadequados para consumo de mesa (Tabela 1). Assim, com exceção destas três variedades, todas as demais avaliadas neste estudo podem ser consideradas de qualidade adequada para consumo de mesa segundo este parâmetro, ao serem colhidas aos

Tabela 1. Tempo de cozimento e resistência ao corte das raízes de mandioca.

\begin{tabular}{|c|c|c|c|c|c|c|c|c|}
\hline \multirow{2}{*}{ Variedade } & \multirow{2}{*}{$\begin{array}{c}\text { Tempo de } \\
\text { Cozimento (min) }\end{array}$} & \multirow{2}{*}{ CV (\%) } & \multicolumn{6}{|c|}{ Resistência ao corte ( $\left.\mathbf{N}^{1}\right)$} \\
\hline & & & Mandioca Crua & $\mathbf{N}$ & CV (\%) & Mandioca Cozida & CV (\%) & $\mathbf{N}$ \\
\hline Aipim Pretinho & $30^{\mathrm{abc}}$ & 3,9 & $22,9^{a}$ & 9 & 16,3 & $1,3^{\mathrm{bcd}}$ & 23,4 & 9 \\
\hline Vermelho Alagoano & $24^{\text {bcde }}$ & 13,2 & $12,2^{\mathrm{dfg}}$ & 7 & 16,1 & $1,6^{b}$ & 39,3 & 9 \\
\hline Pesagro & $24^{\text {cde }}$ & 18,2 & $11,8^{\text {efg }}$ & 8 & 18,9 & $1,1^{\text {bcde }}$ & 35,9 & 9 \\
\hline Cacau Violeira & $24^{\text {cde }}$ & 4,2 & $12,5^{\mathrm{dfg}}$ & 6 & 21,7 & $0,8^{\text {de }}$ & 65,8 & 8 \\
\hline IAC 12 & $25^{\mathrm{abcd}}$ & 2,3 & $13,6^{\text {def }}$ & 8 & 19,8 & $0,8^{\text {cde }}$ & 44,0 & 8 \\
\hline Amarelo Barcelos & $27^{\mathrm{abc}}$ & 2,1 & $15,1^{d}$ & 9 & 13,9 & $3,6^{a}$ & 36,3 & 8 \\
\hline Zumbi & $17^{\dagger}$ & 10,2 & $18,5^{b c}$ & 9 & 24,1 & $1,0^{\text {cde }}$ & 26,3 & 9 \\
\hline Viçosa Martinha & $18^{f}$ & 11,8 & $10,6^{9}$ & 9 & 32,6 & $0,7^{e}$ & 14,1 & 6 \\
\hline BR Gema de Ovo & $19^{\text {def }}$ & 7,9 & $14,4^{\text {de }}$ & 6 & 10,3 & $1,1^{\text {bcde }}$ & 39,3 & 6 \\
\hline IAC 15 & $28^{a b c}$ & 7,3 & $12,0^{\text {efg }}$ & 8 & 16,3 & $3,6^{a}$ & 26,1 & 6 \\
\hline BR Eucalipto & $26^{a b c}$ & 3,8 & $11,3^{\mathrm{fg}}$ & 9 & 16,4 & $1,2^{\text {bcde }}$ & 32,2 & 8 \\
\hline IAC Caapora & $25^{\mathrm{abcd}}$ & 6,0 & $12,5^{\mathrm{dfg}}$ & 8 & 22,1 & $0,7^{e}$ & 46,3 & 7 \\
\hline BR Rosinha & $19^{e f}$ & 13,9 & $15,8^{\mathrm{cd}}$ & 5 & 24,8 & $0,6^{e}$ & 26,4 & 6 \\
\hline IAC Espeto & $31^{a}$ & 3,8 & $10,8^{\mathrm{fg}}$ & 9 & 19,2 & $3,0^{a}$ & 14,4 & 5 \\
\hline IAC 13 & $30^{\mathrm{ab}}$ & 5,0 & $13,1^{\mathrm{dfg}}$ & 8 & 23,5 & $1,6^{b c}$ & 19,3 & 5 \\
\hline
\end{tabular}

${ }^{1}$ Médias com letras iguais na mesma coluna não diferem significativamente a $p \leq 0,05$; Tempo de cozimento: médias obtidas de três mensurações; CV: coeficiente de variação; N: número de repetições. 
onze meses após o plantio. Entretanto, é recomendável a avaliação dessas variedades em outros períodos de colheita, a fim de verificar se os valores ora obtidos se mantêm. Em complementação, é importante que essas raízes sejam avaliadas sensorialmente por consumidores.

Embora o tempo de cozimento seja frequentemente determinado em estudos de raízes de mandioca, não foram encontrados registros dessa variável para as cultivares de abrangência desta pesquisa. Desta forma, em comparação com outros trabalhos, os tempos de cozimento verificados neste experimento foram inferiores ao máximo obtido por Mezette et al. (2009), 52 minutos, e similares às médias observadas por Borges et al. (2002), Lorenzi (1994) e Rimoldi et al. (2006), entre 25 e 29 minutos, e superiores ao mínimo observado por Rimoldi et al. (2005), 15 minutos.

De acordo com Favaro (2003), os fatores responsáveis pelas características de cozimento de mandioca não estão suficientemente esclarecidos. Esse autor constatou diferença no tempo de cozimento entre variedades e entre época de colheita em uma mesma variedade, sendo de mais rápido cozimento as raízes de época de colheita mais precoce.

De fato, vários estudos constataram variação do tempo de cozimento em função da época de colheita, da região de plantio, do genótipo, dentre outros aspectos (ARIAS et al., 2005; RIMOLDI et al., 2005; FUKUDA et al., 2005; SIVIERO et al., 2005; SOUZA et al., 2005; VIEIRA et al., 2009; OLIVEIRA et al., 2010). Entretanto, poucos são aqueles que relatam o coeficiente de variação ou o desvio padrão das medidas. Vale ressaltar que o coeficiente de variação é o desvio padrão expresso como uma porcentagem da dispersão das medidas em relação à média: CV $(\%)=100 \times($ desvio padrão/média).

Os valores de coeficiente de variação para o tempo de cozimento foram satisfatórios e inferiores ao desvio padrão médio observado por Mezette et al. (2009), de 18 minutos para raízes de mesmo clone e de mesma parcela.

Com relação à resistência ao corte da polpa crua, a variedade Viçosa Martinha apresentou a menor média, apesar de não ter diferido estatisticamente $(p>0,05)$ das variedades Cacau Violeira, Pesagro, Vermelho Alagoano, IAC 15, BR Eucalipto, IAC Caapora, IAC Espeto e IAC 13 (Tabela 1). Vale ressaltar que a variedade Viçosa Martinha apresentou o menor tempo de cozimento não diferindo ( $p>0,05)$ das variedades Zumbi e BR Rosinha.

Entretanto, foram as variedades Amarelo Barcelos, IAC 15 e IAC Espeto que apresentaram as maiores médias para resistência ao corte da polpa cozida, enquanto que IAC Caapora, BR Rosinha e Viçosa Martinha, as menores médias, não apresentando diferença significativa ( $p>0,05)$ das variedades BR Eucalipto, Zumbi, BR Gema de Ovo, IAC 12, Pesagro e Cacau Violeira (Tabela 1).
Verificou-se correlação significativa entre a resistência ao corte da polpa cozida e o tempo de cozimento $(0,62)$, indicando que quanto maior a resistência ao corte, maior o tempo de cozimento. Contudo, essa correlação foi moderada, provavelmente em razão dos altos valores de coeficiente de variação calculados para a resistência ao corte. Não foram observadas correlações significativas entre os valores de coeficientes de variação e o número de repetições das medidas de textura, tanto para polpa crua $(r=0,08 ; p=0,7876)$ quanto para polpa cozida $(r=0,44 ; p=0,1012)$. Desta forma, possivelmente a variabilidade dessas medidas tenha ocorrido em função da heterogeneidade das raízes de uma mesma variedade, conforme comentado por Lorenzi (1994). Outros estudos envolvendo avaliação de textura instrumental - porém de alimentos processados - têm considerado coeficientes de variação (CV) elevados, por exemplo, iguais a 40 \%, aceitáveis na avaliação de materiais de características heterogêneas, uma vez que os texturômetros possuem alta sensibilidade (KARAM et al., 2001).

De qualquer forma, outras pesquisas podem ser realizadas visando à otimização da metodologia da avaliação instrumental da textura para raízes de mandioca, uma vez que esta pode tornar-se ferramenta auxiliar no estudo de variedades para as diversas aplicações, além do consumo de mesa.

Comparando-se a variabilidade para ambas as medidas - de tempo de cozimento e de resistência ao corte -, observaram-se menores coeficientes de variação para o tempo de cozimento do que para a resistência ao corte: das 15 variedades avaliadas, apenas quatro apresentaram CV superiores a $10 \%$ para as medidas de tempos de cozimento, enquanto todos se mostraram superiores a esse valor para as medidas de resistência ao corte. Mesmo assim, pode-se verificar, em estudos futuros, se a padronização para único diâmetro das raízes na determinação do tempo de cozimento diminui a variabilidade dessas medidas.

\section{Conclusão}

Nas condições deste trabalho, a medida instrumental de textura não correspondeu a um bom índice da qualidade de raízes de mandioca, em função da heterogeneidade do material e da alta sensibilidade do equipamento. Portanto, a determinação do tempo de cozimento, mesmo utilizando-se um método parcialmente subjetivo (penetração do garfo pelo pesquisador), foi mais adequada para esse fim.

Em função da alta variabilidade observada nas medidas de textura instrumental e tempo de cozimento, este estudo evidencia a importância de se identificar a variedade de raízes de mandioca por ocasião de sua comercialização, à semelhança de outros vegetais. 
Tempo de cozimento e textura de raízes de mandioca

TALMA, S. V. et al.

Em complementação, é importante que essas raízes sejam avaliadas sensorialmente por consumidores.

\section{Agradecimentos}

A equipe agradece à Coordenação de Aperfeiçoamento de Pessoal de Nível Superior (CAPES) pela concessão da Bolsa de Estudos.

\section{Referências}

ARIAS, E. R. A.; ARIAS, S. M. S.; MARTINS, C. S.; PEREIRA, F. A. R.; OTSUBO, A. A. Avaliação da produtividade, tempo de cozimento e padrão de massa cozida de oito cultivares de mandioca tipo mesa, em Campo Grande, MS. In: CONGRESSO BRASILEIRO DE MANDIOCA, 11., 2005, Campo Grande. Resumos... Campo Grande: Embrapa Agropecuária Oeste, 2005.

BORGES, M. F.; FUKUDA, W. M. G. Teor de cianeto em raízes frescas e processadas de mandioca (Manihot esculenta Crantz) de mesa. Revista Brasileira de Mandioca, Cruz das Almas, v. 8, n. 2, p. 71-76, 1989.

BORGES, M. F.; FUKUDA, W. M. G.; ROSSETTI, A .G. Avaliação de variedades de mandioca para consumo humano. Pesquisa Agropecuária Brasileira, Brasília, v. 37, n. 11, p. 1559-1565, 2002. http://dx.doi.org/10.1590/S0100-204X2002001100006

BORGES, M. F.; CARVALHO, V. D.; FUKUDA, W. M. G. Efeito de tratamento térmico na conservação pós-colheita de raízes de mandioca (Manihot esculenta Crantz) de mesa. Revista Brasileira de Mandioca, Cruz das Almas, v. 11, n. 1, p. 7-18, 1992.

COMPANHIA DE ENTREPOSTOS E ARMAZÉNS GERAIS DE SÃO PAULO - CEAGESP. Seção de Economia e Desenvolvimento. Sazonalidade dos Produtos Comercializados no ETSP. São Paulo: CEAGESP, 2011. Disponível em: <http://www.ceagesp. gov.br/produtos/epoca/produtos_epoca.pdf>. Acesso em: 27 jan. 2011.

FAVARO, S. P. Composição Química e Estrutura de Paredes Celulares de Variedades de Mandioca (Manihot esculenta Crantz) com Tempos de Cocção Diferentes. 2003. $132 \mathrm{f}$. Tese (Doutorado em Ciência e Tecnologia de Alimentos)-Universidade Estadual de Londrina, Londrina, Paraná, 2003.

FENNEMA, O. R. Química de los Alimentos. Zaragoza: Acríbia, 1992. $1095 \mathrm{p}$.

FOlegatTI, M. I. S.; MATSUURA, F. C. A. U. Mandioca e derivados. Cruz das Almas: Embrapa-CNPMF, [1997]. Disponível em: <http://www.embrapa.br>. Acesso em: 21 jan. 2011.

FONTES, P. S. F. Adubação Nitrogenada e Avaliação de Cultivares de Banana (Musa spp.) no Noroeste do Estado do Rio de Janeiro. 2002. 64 f. Dissertação (Mestrado em Produção
Vegetal)-Universidade Estadual do Norte Fluminense, Campos dos Goytacazes, Rio de Janeiro, 2002.

FOOD AND AGRICULTURE ORGANIZATION - FAO. Base de Dados de 2010. Roma: FAO, 2010. Disponível em: <http://www. fao.org>. Acesso em: 25 jan. 2011.

FUKUDA, W. M. G.; BORGES, M. F. Avaliação qualitativa de cultivares de mandioca de mesa. Revista Brasileira de Mandioca, Cruz das Almas, v. 7, n. 1, p. 63-71, 1988.

FUKUDA, W. M. G.; BORGES, M. F. Influência da idade de colheita sobre a qualidade de raízes em diferentes cultivares de mandioca de mesa. Revista Brasileira de Mandioca, Cruz das Almas, v. 9, p. 7-19, 1990.

FUKUDA, W. M. G.; PEREIRA, M. E. C.; FOLLEGATTI, M. L. S. Efeito da idade de colheita sobre a qualidade, produtividade e teor de carotenóides em raízes de variedades de mandioca para mesa. In: CONGRESSO BRASILEIRO DE MANDIOCA, 11., 2005, Campo Grande. Resumos... Campo Grande: Embrapa Agropecuária Oeste, 2005.

KARAM, L. B.; GROSSMANN, M. V. E.; SILVA, R. S. S. F. Misturas de farinha de aveia e amido de milho com alto teor de amilopectina para produção de "snacks". Ciência e Tecnologia Alimentos, São Paulo, v. 21, n. 2, p. 158-163, 2001. http://dx. doi. org/10.1590/S0101-20612001000200007

LAWLESS, H. T.; HEYMANN, H. Sensory Evaluation of Food: Principles and Practices. Gaithersburg: Aspen Publishers, 1999. 827 p. (Series III).

LORENZI, J. O. Variação na qualidade culinária das raízes de mandioca. Bragantia, Campinas, v. 53, n. 2, p. 237-245, 1994. http://dx.doi.org/10.1590/S0006-87051994000200013

LORENZI, J. O. Mandioca. Campinas: CATI, 2003. 116 p. (Boletim Técnico, n. 245).

MATTOS, P. L. P.; SOUZA, L. S.; CALDAS, R. C.; PORTO, M. C. M. Adaptação de espaçamentos em fileiras duplas para a cultura da mandioca (Manihot esculenta Crantz). In: CONGRESSO BRASILEIRO DE MANDIOCA, 1., 1979, Cruz das Almas. Anais... Cruz das Almas: Embrapa Agropecuária Oeste, 1979. p. 19-34.

MENOLI, A. V.; BELEIA, A. Starch and pectin solubilization and texture modification during pre-cooking and cooking of cassava root (Manihot esculenta Crantz). Food Science and Technology, Amsterdam, v. 40, n. 4, p. 744-747, 2007

MEZETTE, T. F.; CARVALHO, C. R. L.; MORGANO, M. A.; SILVA, M. G.; PARRA, E. S. B.; GALERA, J. M. S. V.; VALLE, T. L. Seleção de clones-elite de mandioca de mesa visando a características agronômicas, tecnológicas e químicas. Bragantia, Campinas, v. 68 , n. 3, p. 601-609, 2009. http://dx.doi.org/10.1590/S000687052009000300006

OLIVEIRA, S. P; VIANA, A. E. S.; MATSUMOTO, S. N.; CARDOSO JÚNIOR, N. S.; SEDIYAMA, T.; SÃO JOSÉ, A. R. Efeito da poda e de épocas de colheita sobre características agronômicas da 
mandioca. Acta Scientiarum. Agronomy, Maringá, v. 32, n. 1, p. 99-108, 2010.

OSIRU, D. S. O.; HAHN, S. K.; OSONUBI, O. Varietal response to drought stress in cassava. In: INTERNATIONAL SOCIETY FOR TROPICAL ROOT CROPS - ISTRC. Tropical Root Crops. Ibadan: IITA, 1992. p. 97-102.

PADONOU, W.; MESTRES, C.; NAGO, M. C. The quality of boiled cassava roots: instrumental characterization and relationship with physicochemical properties and sensorial properties. Food Chemistry, London, n. 89, p. 261-270, 2005. http://dx.doi. org/10.1016/j.foodchem.2004.02.033

RIMOLDI, F.; VIDIGAL FILHO, P. S.; CLEMENTE, E.; VIDIGAL, M. C. G.; MELO, J. M.; ZANATTA, C. L. Z.; KVITSCHAL, M. V. Teores de amido, de $\mathrm{HCN}$ e tempo de cozimento de raízes tuberosas de cultivares de mandioca de mesa coletadas no Paraná. In: CONGRESSO BRASILEIRO DE MANDIOCA, 11., 2005, Campo Grande. Resumos... Campo Grande: Embrapa Agropecuária Oeste, 2005.

RIMOLDI, F.; VIDIGAL FILHO, P. S.; VIDIGAL, M. C. G.; CLEMENTE, E.; PEQUENO, M. G.; MIRANDA, L.; KVITSCHAL, M. V. Produtividade, composição química e tempo de cozimento de cultivares de mandioca de mesa coletadas no Estado do Paraná. Acta Scientiarum. Agronomy, Maringá, v. 28, n. 1, p. 63-69, 2006.
SIVIERO, A.; SOUZA, J. M. L.; MENDONÇA, H. A.; ALVERGA, P. P. 'Caipora' e 'Mani': cultivares de mandioca de mesa para o Acre. In: CONGRESSO BRASILEIRO DE MANDIOCA, 11., 2005, Campo Grande. Resumos... Campo Grande: Embrapa Agropecuária Oeste, 2005.

SOUZA, J. M. L.; SIVIERO, A.; MENDONÇA, H. A.; REIS, F. S. Caracterização de raízes e da qualidade da massa de genótipos de mandioca para consumo in natura no estado do Acre. In: CONGRESSO BRASILEIRO DE MANDIOCA, 11., 2005, Campo Grande. Resumos... Campo Grande: Embrapa Agropecuária Oeste, 2005.

VIEIRA, E. A.; FIALHO, J. F.; SILVA, M. S.; FUKUDA, W. M. G.; SANTOS FILHO, M. O. S. Comportamento de genótipos de mandioca de mesa no Distrito Federal. Revista Ciência Agronômica, Fortaleza, v. 40, n. 1, p. 113-122, 2009.

WHEATLEY, C.; GOMEZ. G. Evaluation of some quality characteristics in cassava storage roots. Qualitas Plantarum Plant Foods for Human Nutrition, The Hage, v. 35, n. 2, p. 121-129, 1985. http://dx.doi.org/10.1007/BF01092127

WHEATLEY, C. C. Conservación de Raíces de Yuca en Bolsas de Polietileno. Cali: Centro Internacional de Agricultura Tropical, $1987.33 \mathrm{p}$. 